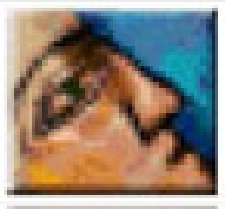

Diálogos Revista Electrónica de Historia

E-ISSN: 1409-469X

historia@fcs.ucr.ac.cr

Universidad de Costa Rica

Costa Rica

Alfaro, Eric J.; Quesada, Adolfo; Solano, Flora

ANÁLISIS DEL IMPACTO EN COSTA RICA DE LOS CICLONES TROPICALES OCURRIDOS EN EL

MAR CARIBE DESDE 1968 AL 2007

Diálogos Revista Electrónica de Historia, vol. 11, núm. 2, septiembre-febrero, 2010, pp. 26-38

Universidad de Costa Rica

San Pedro de Montes de Oca, Costa Rica

Disponible en: http://www.redalyc.org/articulo.oa?id=43922144002

- Cómo citar el artículo

- Número completo

- Más información del artículo

- Página de la revista en redalyc.org

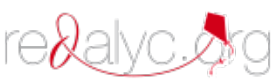

Sistema de Información Científica

Red de Revistas Científicas de América Latina, el Caribe, España y Portugal

Proyecto académico sin fines de lucro, desarrollado bajo la iniciativa de acceso abierto 
Palabras claves

Huracanes, Ciclones Tropicales, Impactos, Desastres Naturales, Mar Caribe, América Central, Variabilidad Climática.

Keywords

Hurricanes, Tropical Cyclones, Impacts, Natural Disasters, Caribbean Sea, Central America, Climate Variability.

Fecha de recepción: 15 de abril 2008 - Fecha de aceptación: 16 de setiembre 2010

\section{Resumen}

El estudio de la actividad de los ciclones tropicales, así como su caracterización histórica en la región centroamericana, es una prioridad para apoyar las actividades de mitigación del impacto que estos fenómenos provocan en las diversas zonas del istmo. Basado en lo anterior, en este trabajo se definen para Costa Rica, los años de alto o bajo impacto por el paso de ciclones tropicales en el Caribe considerando variables asociadas a la trayectoria del evento, la permanencia del mismo cerca del istmo y la frecuencia de los fenómenos en la cuenca. El análisis mostró que la mayor probabilidad de ocurrencia de los ciclones tropicales cerca de América Central se presenta durante el trimestre agosto-setiembre-octubre. Se observó también, que durante los años que mostraron altas ocurrencias de ciclones cerca de América Central, el Atlántico Tropical Norte presentó campos con anomalías positivas en la temperatura superficial del mar y la humedad relativa en $700 \mathrm{hPa}$, y negativas en la presión media al nivel del mar. Además, Costa Rica posee una rica fuente en información sobre desastres de origen natural que afectaron directamente centros poblacionales y diversos sectores socio-económicos, como el agropecuario, energía y transporte, entre otros. Esto permitió el detalle de los desastres sufridos en Costa Rica por ciclones tropicales durante las últimas cuatro décadas. El trabajo indicó que la tendencia encontrada en el reporte anual de impactos por eventos hidrometeorológicos, no puede ser explicada totalmente por efectos climáticos, lo que hace necesario incluir variables de otro tipo, como las socio-económicas.

The study of tropical cyclones activity in the Caribbean and their historic characterization in the Central American region is a basic element to mitigate their impact over different regions of the isthmus. Years with high and low tropical cyclone impacts in Central America were defined, considering variables like track, residence time near the isthmus and the annual occurrence of cyclones in the basin. The analysis showed that tropical cyclones are more likely to occur near Central America during August-September-October. Also, positive sea surface temperature and relative humidity at $700 \mathrm{hPa}$ and negative sea level pressure anomalies at the Tropical North Atlantic were observed for the years that showed high occurrences of tropical cyclones near Central America. Additionally, Costa Rica has a good natural disasters data base, related with events that affected specific locations and several socioeconomic sectors like agriculture, energy and transport. This data base, allowed the study of disasters in Costa Rica related with tropical cyclones during the last four decades. The analysis showed a trend in the annual number of impacts related with hydro-meteorology causes that cannot be explained by climate trends only. That means that other variables like those related with socioeconomic aspects should be included in the analysis to explain this variability and their associated impacts.

Eric J. Alfaro: Centro de Investigaciones Geofísicas, Escuela de Física, Centro de Investigación en Ciencias del Mar y Limnología, Universidad de Costa Rica

Adolfo Quesada : Centro de Investigaciones Geofísicas

Flora Solano : Centro de Investigaciones Geofísicas 


\title{
ANÁLISIS DEL IMPACTO EN COSTA RICA DE LOS CICLONES TROPICALES OCURRIDOS EN EL MAR CARIBE DESDE 1968 AL 2007
}

\author{
Eric J. Alfaro \\ Adolfo Quesada \\ Flora Solano
}

\section{INTRODUCCIÓN}

El estudio del impacto de ciclones tropicales en América Central es recurrente $^{1}$. El bajo desarrollo social, así como el alto riesgo de las poblaciones elevan la vulnerabilidad de la región ante el impacto de estos eventos. Por lo tanto, el abordaje de la dinámica propia de los ciclones tropicales debe darse ligado a los estudios de los impactos que éstos provocan en la sociedad, para entender bajo qué circunstancias sus efectos son mayores ${ }^{2}$.

De especial interés resulta lo expuesto en diferentes comunicaciones relacionadas con el cambio climático, al plantear un incremento en la ocurrencia y en la intensidad de dichos ciclones en la cuenca del Atlántico Norte, una de las principales cuencas ciclogenéticas del mundo ${ }^{3}$. No obstante, el tema es aún de amplio debate en la literatura científica, ya que algunos estudios plantean una disminución en el número de ciclones tropicales en el Atlántico ante un escenario de cambio climático ${ }^{4}$. Por otro lado, dependiendo del período y de la variable asociada a los ciclones tropicales en estudio, se cuestiona o no el incremento que se observa en el pasado ${ }^{5}$. Según Neumann et al. (1993), en la década de 1940 se iniciaron los vuelos militares de reconocimiento para la recolección de datos in situ de los ciclones tropicales, por lo que los datos de tormentas ausentes anteriores a ese periodo hacen que los análisis de los aspectos asociados a los ciclones tropicales en el Atlántico, sean poco confiables cuando se utilizan como series de tiempo.

Independientemente del tema del incremento o no de ciclones tropicales en el Atlántico, éstos han mostrado periodos de gran variabilidad en el pasado ${ }^{6}$ y mucho del incremento en la actividad ciclónica se explica por una variabilidad multidecadal de la temperatura superficial del mar presente en el Océano Atlántico, el cual ha venido presentando condiciones cálidas en los últimos años ${ }^{7}$. Por otro lado, los impactos tanto en la parte social como económica en la región de la cuenca del Caribe son apreciables debido a su elevada condición de vulnerabilidad ante los desastres de origen natural ${ }^{8}$. Por lo anterior, se plantea como objetivo de este trabajo, el estudiar las ocurrencias de ciclones tropicales cerca de América Central y los reportes de impactos por desastres asociados a eventos hidrometeorológicos 
y relacionados con ciclones tropicales en Costa Rica en las últimas cuatro décadas. Además, se explorará su variabilidad y la posible relación entre ambas. Los datos y metodología empleados se exponen en la sección 2 . Los resultados y su discusión en el apartado 3 y por último, en la sección 4 las principales conclusiones de este trabajo.

\section{DATOS Y METODOLOGÍA}

El periodo de tiempo analizado en este trabajo fue de 1968 a 2007 (40 años). Este periodo fue escogido simplemente para que concordara con la base de datos de desastres que se detallará más adelante. La base de datos con información sobre los ciclones tropicales utilizada corresponde a la del proyecto HURDAT ${ }^{9}$.

Esta base de datos es pública y se accede en la dirección http://www.aoml. noaa.gov/hrd/hurdat $/{ }^{10}$. Contiene datos relacionados con el ciclón tropical cada seis horas y de las variables: fecha, posición geográfica, dirección del ciclón, velocidad del ciclón, velocidad del viento en la pared del ojo del ciclón, presión atmosférica en el ojo del ciclón y la categoría del mismo, según la escala de SaffirSimpson ${ }^{11,12}$. Las categorías que abarca está base de datos van desde perturbación tropical hasta huracán categoría 5 y todos los sistemas en este trabajo fueron clasificados de la misma manera.

Como primer paso se extrajo de la base de datos la información correspondiente a aquellos ciclones tropicales que cruzaron o se formaron en o cerca del Mar Caribe; es decir, con posiciones geográficas observadas menores a $24^{\circ}$ de latitud norte y mayores a $60^{\circ}$ de longitud oeste. Para estudiar la ocurrencia de estos fenómenos en el Mar Caribe se formaron cuatro series de tiempo anuales. La primera corresponde a la suma total de ciclones tropicales, la segunda para las tormentas con nombre, las cuales se agrupan en tormentas tropicales y huracanes; la tercera la componen sólo los ciclones tropicales catalogados como huracán, y la cuarta de huracanes identificados como intensos, es decir aquellos que alcanzaron categoría tres o superior. Posteriormente, se determinaron los años con mayor o menor ocurrencia de ciclones tropicales en el Mar Caribe.

Para estudiar la ocurrencia de estos ciclones tropicales cerca de la costa caribe de América Central, se creó un índice anual que consiste en la suma de las todas las posiciones encontradas dentro de un círculo de radio de 3 o $7^{\circ}$ con respecto a algún punto de la costa, desde $89^{\circ} \mathrm{W}-21.5^{\circ} \mathrm{N}$ a $77^{\circ} \mathrm{W}-8.3^{\circ} \mathrm{N}$ (Fig. 1). Posteriormente se sumaron las posiciones encontradas en todos los círculos, en forma similar al criterio usado por Alvarado y Alfaro (2003) para Puerto Limón en Costa Rica. Este índice surgió debido a que se ha observado que la magnitud del impacto de los ciclones tropicales sobre un sitio de interés depende de variables, tales como: la posición del ciclón respecto a ese sitio y/o el tiempo de permanencia 
del mismo cerca del área de estudio o velocidad de avance ${ }^{13}$. Al igual que para las series anteriores, se cuantificaron los años que presentaron mayor o menor presencia de ciclones tropicales con respecto a la costa caribe de América Central. Tomando en cuenta estos datos, se hicieron mapas compuestos de anomalías de distintas variables geofísicas que consisten en la diferencia del promedio de los años con mayor ocurrencia con respecto a aquellos de menor ocurrencia durante el trimestre agosto, setiembre y octubre (ASO), meses que típicamente presentan una mayor frecuencia de ciclones tropicales en el Océano Atlántico ${ }^{14}$. Las variables estudiadas son la Temperatura Superficial del Mar (TSM), la Presión Atmosférica Media al Nivel del Mar (PMNM) y de la Humedad Relativa en el nivel de $700 \mathrm{hPa}$ (HR700), ya que según Latif et al. (2007), DeMaria et al. (2001), Hobgood (2005) y Lizano y Fernández (1996), la formación de los ciclones tropicales en los océanos se ve favorecida cuando la temperatura de la capa superficial supera los $26^{\circ} \mathrm{C}$, aunado a la existencia de una zona de baja presión atmosférica y una alta humedad en la troposfera media $(700 \mathrm{hPa})$. Para tal fin, se utilizaron los datos del reanálisis del NCEP/NCAR (Kalnay et al., 1996) y los datos concatenados de los análisis de Kaplan et al. (1998) y Reynolds y Smith (1994) para preparar mapas compuestos de las anomalías de diferentes variables troposféricas y de TSM para los años con alta o baja ocurrencia de ciclones cerca de América Central.

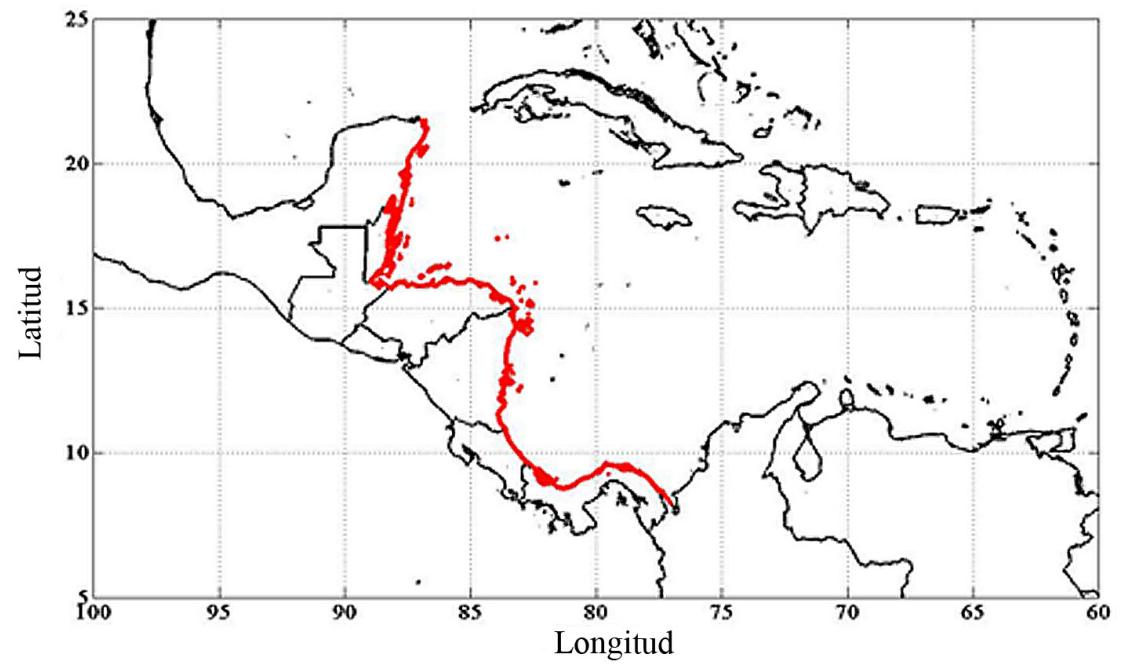

Figura 1. En rojo se presenta la costa Caribe de América Central que fue considerada para estudiar la ocurrencia de los ciclones tropicales, ésta abarcó desde $89^{\circ} \mathrm{W}-21.5^{\circ} \mathrm{N}$ en Yucatán hasta $77^{\circ} \mathrm{W}-8.3^{\circ} \mathrm{N}$ en el tapón del Darién (datos disponibles en http://rimmer.ngdc.noaa.gov/, última visita 22/01/2010). 
La información sobre los reportes de desastres durante este periodo se obtuvo de la base de datos DesInventar ${ }^{15}$, esta base de datos es pública y se accede en el sitio http://www.desinventar.org (última visita el 18 de enero de 2010). De ésta se extrajo información anual para Costa Rica de los desastres ocasionados por eventos hidrometeorológicos, exceptuando aquellos definidos como sequías ${ }^{16}$. Posteriormente, se seleccionaron los registros asociados a las fechas en que se reportó la presencia de algún ciclón tropical en la cuenca del Mar Caribe. Lo anterior produjo dos series anuales de la suma de los reportes encontrados: la de todos los eventos hidrometeorológicos y la de sólo los relacionados con la presencia de algún ciclón tropical. Esta base de datos tiene reportes desde 1968, no obstante, los reportes hidrometeorológicos se iniciaron a partir del año 1970. Actualmente se trabaja en la recopilación y reconstrucción de la información de la ocurrencia de desastres naturales para los dos años iniciales a partir de datos periodísticos ${ }^{1718}$.

La información obtenida de DesInventar fue estudiada y desagregada en el nivel nacional, cantonal (municipio, segunda unidad administrativa) y distrital (tercera unidad administrativa). Estos datos fueron contrastados posteriormente con el Índice de Desarrollo Social (IDS), elaborado por el Ministerio de Planificación de Costa Rica en el año 2007 por el Ministerio de Planificación de Costa Rica ${ }^{18}$. Por último, para explorar posibles relaciones entre el impacto de ciclones tropicales y alguna variable macroeconómica en Costa Rica, se estudió la tasa de variación del Producto Interno Bruto (PIB), a precios de mercado, en función de la ocurrencia de ciclones tropicales cercanos a la costa caribe de América Central. La información del PIB fue obtenida del Banco Central de Costa Rica (ver http://www.bccr.fi.cr, última visita 22/02/2010).

\section{RESULTADOS Y DISCUSIÓN}

En la Fig. 2 se muestran las series anuales de la ocurrencia de ciclones tropicales en el Mar Caribe. Se observa una tendencia lineal positiva en todas las series de esta figura, sin embargo sólo la correspondiente a la de los huracanes intensos muestra una tendencia con un valor- $p$ asociado menor a 0.010. Las series de los huracanes y de las tormentas con nombres muestran valores- $p$ mayores a 0.010 pero menores a $0.050 \mathrm{y}$ el correspondiente al total de los ciclones tropicales fue mayor a 0.100 . Esto último, significa que no se tiene evidencia estadística suficiente durante este periodo, para afirmar que el número total de ciclones tropicales está aumentando en el Mar Caribe, de acuerdo a la definición empleada. Lo anterior concuerda parcialmente con lo hallado por Alfaro (2007), quién observó para el período 1944-2004, que el número 
anual del total de ciclones tropicales en el Atlántico mostraba una tendencia lineal positiva significativa, principalmente debido a que la tendencia asociada a la serie de tormentas tropicales era también positiva y significativa. Esto también explica el valor observado para la serie del total de los ciclones menores (tormentas tropicales y huracanes de categoría 2 y 3 ), mientras que para ninguna de las series de huracanes se observó alguna tendencia significativa. Esto evidencia lo expuesto por Landsea et al. (2009), Kunkel (2008), Kossin et al. (2007) y Landsea et al. (2006) quienes señalan que los estudios de tendencias sobre ciclones tropicales en el Atlántico se pueden modificar en función de la variable y el periodo de tiempo escogido para el análisis, tal y como encontró Klotzbach (2006), quién reportó que sus datos indican una tendencia de gran aumento en la intensidad y la longevidad de los ciclones tropicales en la cuenca del Atlántico Norte. En este análisis, es importante recordar que el año 2005 fue uno particularmente activo, lo cual puede afectar el estudio de las tendencias ${ }^{19}$.

Para el periodo analizado 1968-2007, el número total de ciclones tropicales fue de 208, de los cuales 174 fueron tormentas con nombre, 88 huracanes y 43

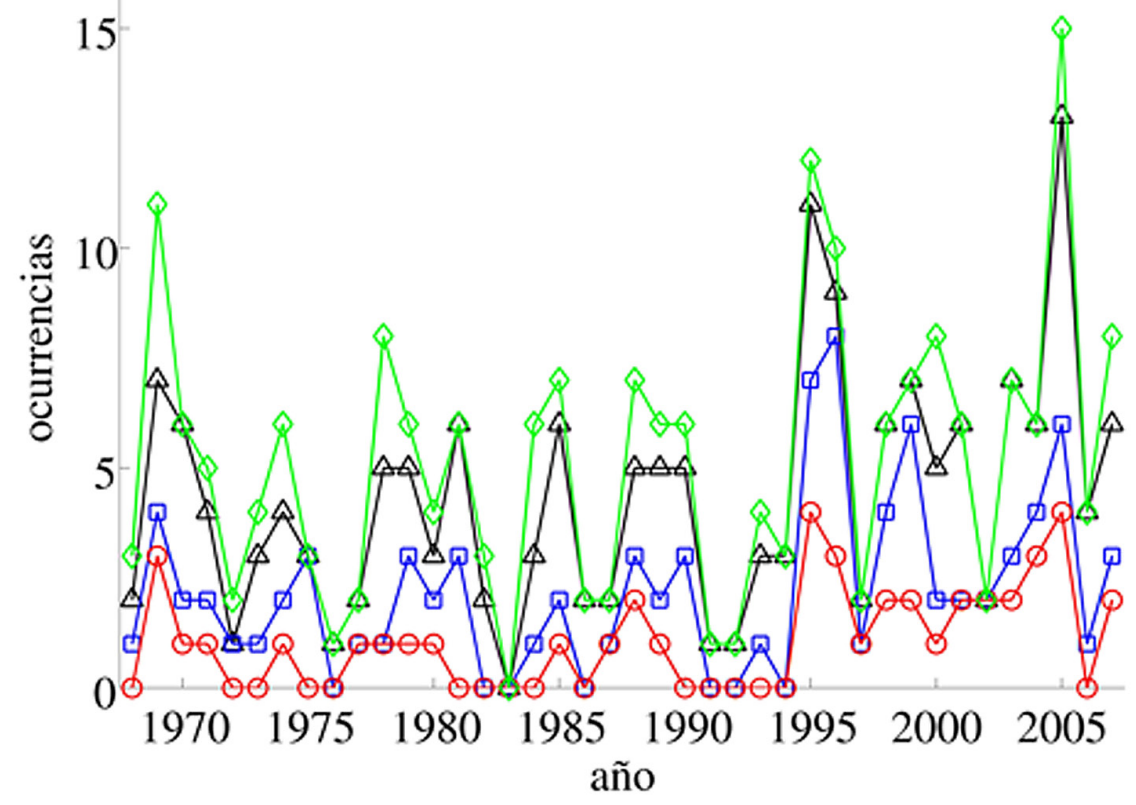

Figura 2. Series anuales de la ocurrencia de ciclones tropicales en el Mar Caribe (ocurrencia en latitudes menores a $24^{\circ} \mathrm{N}$ y longitudes mayores a $60^{\circ} \mathrm{W}$ ), a partir de la base de datos HURDAT para 1968-2007. La línea verde con rombos es para el total de ciclones tropicales, la negra con triángulos es para el total de las tormentas con nombre, la azul con cuadros para el total de huracanes y la roja con círculos para el total de huracanes intensos (categoría 3 o mayor). Las tendencias (valores- $p$ ) observadas(os) durante este período fueron de 0.07 (0.109), $0.09(0.017), 0.05(0.041)$ y $0.04(0.006)$ eventos año ${ }^{-1}$, respectivamente. 
huracanes intensos. La mediana (intervalo intercuartil) de cada tipo fue de: 6 (4.5), $4(4.0), 2$ (2.0) y 1 (2.0), respectivamente. Para la serie de tiempo asociada con el total de los ciclones tropicales, los años identificados con altas ocurrencias fueron: 2005 con 15 eventos, 1995 con 12, 1969 con 11, 1996 con 10, y 2007, 2000 y 1978 con 8 eventos, respectivamente. Los años identificados con bajas ocurrencias fueron: 1983 que no presentó ningún evento, 1976, 1991, 1992 con un evento, y 1972, 1977, 1986, 1987, 1997 y 2002 con 2 eventos.

La Tabla 1, muestra los años con más y menos posiciones cercanas a la costa del Caribe de América Central dentro de un radio de $3^{\circ}$ con respecto a algún punto de la costa. A modo de comparación se realizó este mismo ejercicio en las costas de las Antillas y del Golfo de México. Se destaca que no necesariamente un año con mayor o menor frecuencia cerca de la costa caribe de América Central, coincide con un año de mayor o menor frecuencia cerca de las Antillas o la costa del Golfo de México. Nótese que de los años observados con pocas ocurrencias cerca de América Central coinciden 6 (5) de 8 con los observados cerca de las Antillas (del Golfo de México) y sólo 3 de 8 son los mismos para las tres regiones (1976, 1986 y 1992), mientras que para los años con mayor ocurrencia 2 (3) de 8 de ellos coinciden con los observados cerca de las Antillas (del Golfo de México) y sólo el 2005 coincide para las tres regiones.

Sin embargo, en América Central muchos de los efectos provocados por los ciclones tropicales se deben a los efectos llamados "indirectos" 20 ; es decir, debido a la circulación inducida por el ciclón sobre la región en la cual provoca un flujo de aire caliente y húmedo, proveniente del Pacífico, que al encontrarse la cordillera produce precipitación de tipo estratiforme a consecuencia del ascenso por forzamiento orográfico sobre la Vertiente del Pacífico de América Central. Este tipo de eventos algunas veces son llamados "temporales" 21 . Debido a lo anterior, en este trabajo se amplió el criterio a $7^{\circ}$ de radio desde algún punto de la costa caribe de América Central para encontrar las posiciones cercanas al istmo, siguiendo lo sugerido por Alvarado y Alfaro (2003). La Fig. 3, muestra el índice normalizado para este nuevo umbral de $7^{\circ}$. Este índice mostró una tendencia lineal positiva pero con un valor- $p$ asociado mayor a 0.100 , lo que significa que no se tendría evidencia estadística suficientemente fuerte para decir que está ocurriendo una mayor presencia de ciclones tropicales cercanos a las costas de América Central. Los años identificados con mayores ocurrencias cerca de la costa fueron: 1969, 1970, 1971, 1995, 1996, 1998, 2001 y 2005. Los años con menores ocurrencias fueron: 1976, 1982, 1983, 1984, 1986, 1991, 1992 y 1997. En ambos casos, seis de los años anteriores coinciden con los mostrados en la Tabla 1 para América Central. La correlación de Pearson, entre esta serie y la de la frecuencia anual del total de ciclones tropicales en el Mar Caribe mostrada en la Fig. 2 es de 0.77 , con una significancia estadística superior al $99 \%{ }^{22}$. El evento 
identificado como de mayor impacto por este índice fue el huracán Mitch en el año 1998.

La Fig. 4 presenta las posiciones registradas durante los años de mayor (a) y menor ocurrencia (b). Nótese, que la metodología empleada diferencia aquellos años para los que se registraron más ciclones cerca de la costa caribe de América Central, de aquellos los cuales prácticamente no se registraron ciclones cercanos. La Fig. 5 muestra el número de posiciones encontradas por mes cerca de la costa caribe de América Central. Se observa que durante el trimestre ASO, es cuando es más probable $(69 \%)$ encontrar ciclones tropicales dentro de un radio de $7^{\circ}$ cerca de la costa, lo que coincide también con los meses de mayor frecuencia de Ciclones Tropicales en el Caribe ${ }^{23}$.

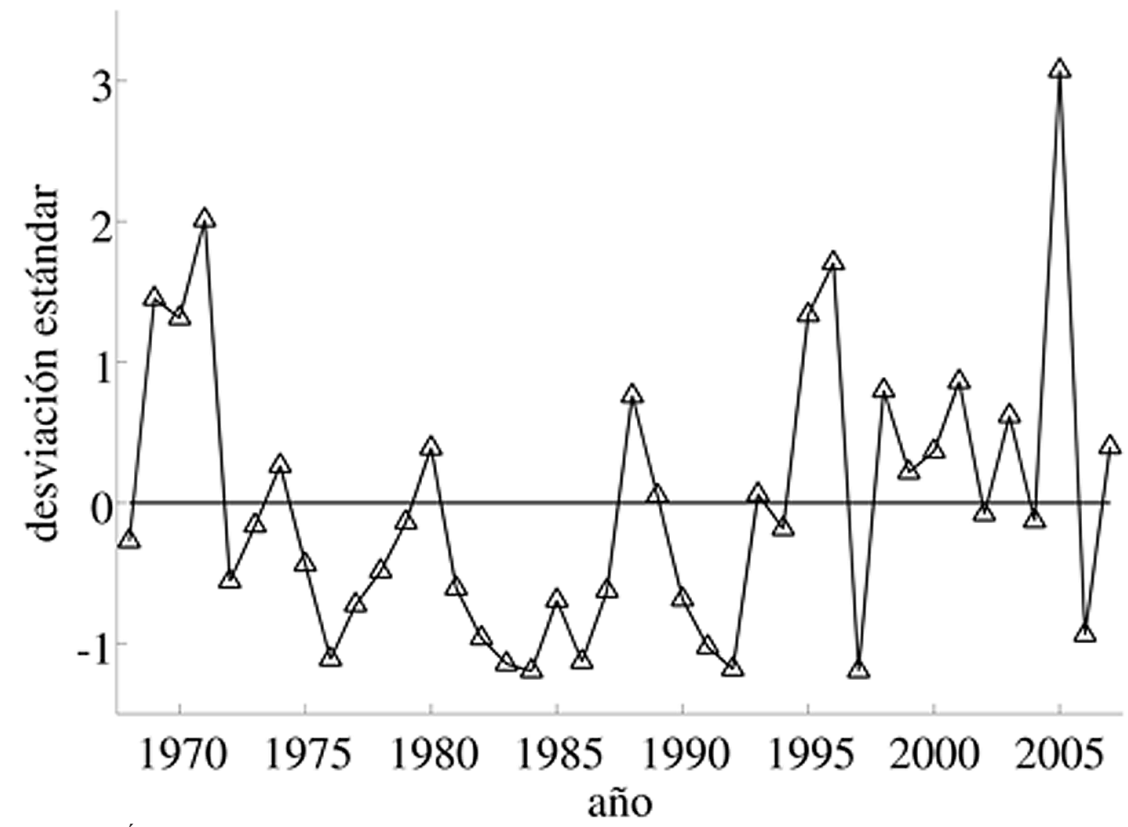

Figura 3. Índice normalizado de ocurrencias de ciclones tropicales dentro de un radio de $7^{\circ}$ a partir de algún punto de la costa caribe de América Central observadas en la base de datos HURDAT. Este índice mostró una tendencia lineal positiva de $1.13 \times 10^{-2}$ año $^{-1}$, con un valor-p asociado de mayor a 0.415 . 
(a)

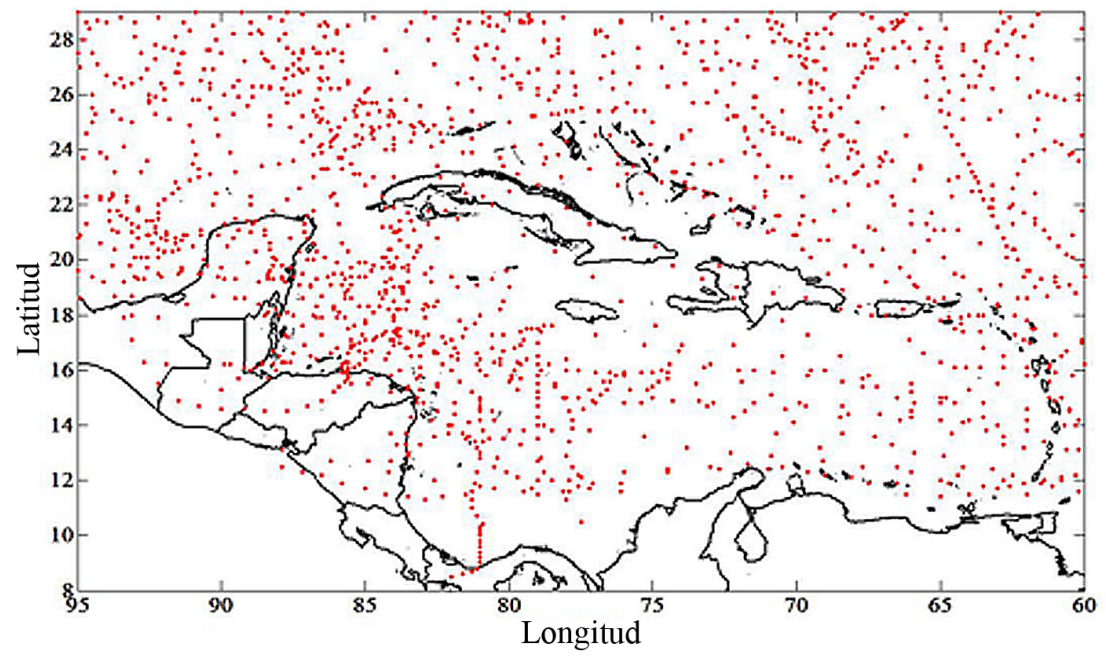

(b)

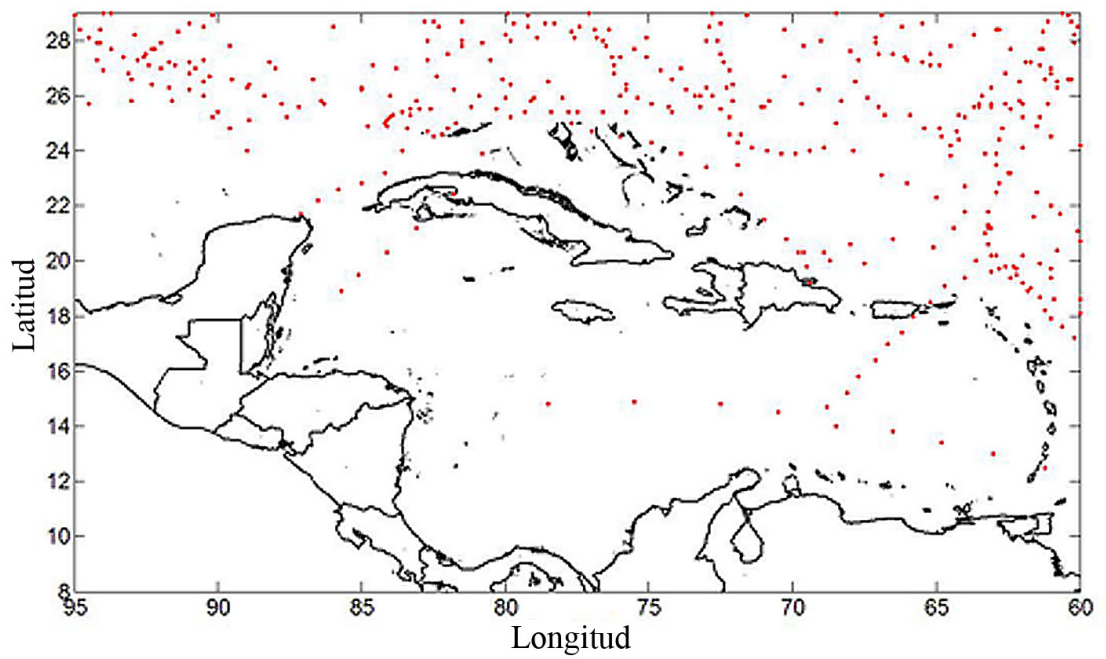

Figura 4. Posiciones registradas (puntos rojos) en la base de datos HURDAT durante los años de mayor (a) y menor (b) ocurrencia. De acuerdo a la Fig. 3, los años identificados con mayor ocurrencia cerca de la costa fueron: 1969, 1970, 1971, 1995, 1996, 1998, 2001 y 2005. Los años con menor ocurrencia fueron: 1976, 1982, 1983, 1984, 1986, 1991, 1992 y 1997. 


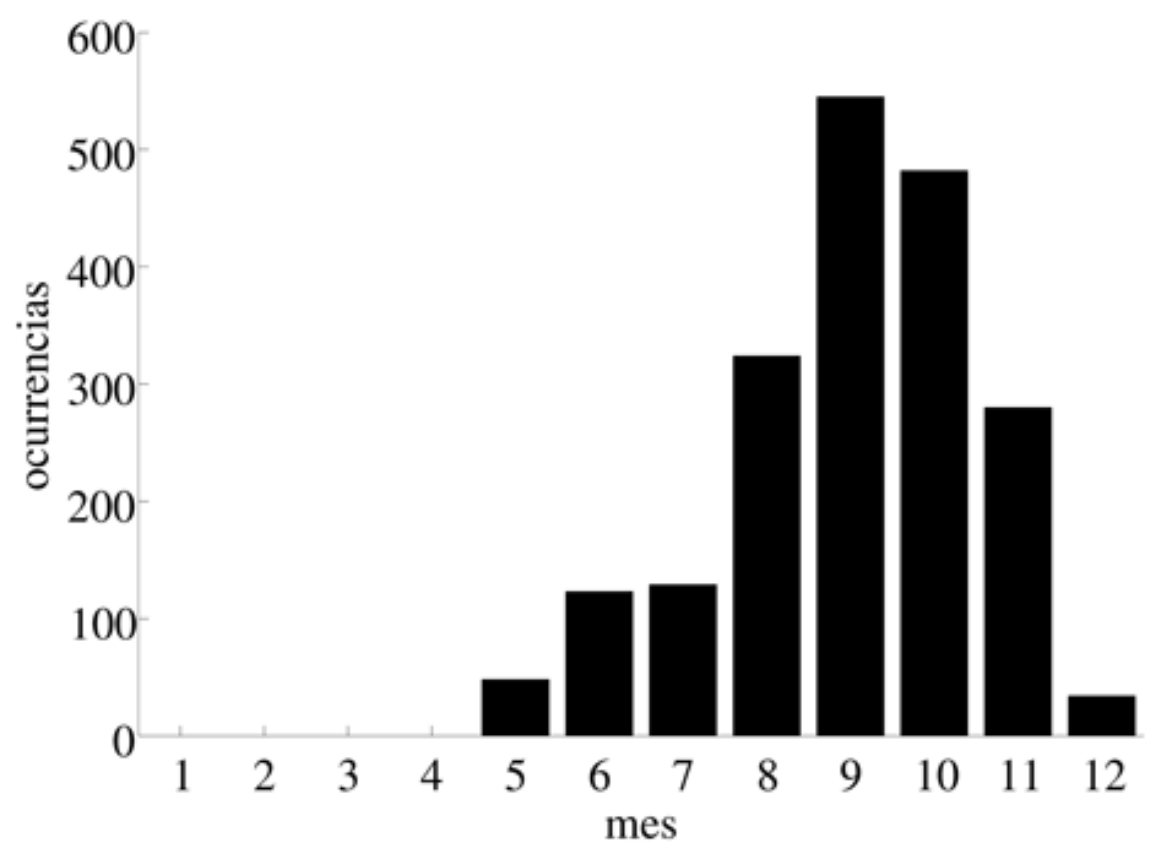

Figura 5. Número de posiciones encontradas por mes en la base de datos HURDAT dentro de un radio de $7^{\circ}$ a partir de algún punto de la costa Caribe de América Central.

Los mapas compuestos de la Temperatura Superficial del Mar(TSM), la Presión Atmosférica Media al Nivel del Mar (PMNM) y la Humedad Relativa en el nivel de $700 \mathrm{hPa}$ (HR700) se presentan en la Fig. 6. Estos mapas consisten en la diferencia del promedio de las anomalías de aquellos años con mayor ocurrencia con respecto a aquellos años de menor ocurrencia durante el trimestre de ASO. Nótese, que al comparar los años de mayor ocurrencia con respecto a los de menor ocurrencia se observan anomalías positivas de TSM en la región del Océano Atlántico tropical y anomalías negativas en el Pacífico ecuatorial, asociado con el fenómeno de El Niño-Oscilación del Sur (ENOS, Fig. 6a). El Atlántico Tropical también muestra una región con anomalías negativas en el campo de la PMNM y positivas en la HR700 (Figs. 6b y c, respectivamente). Según describe Alfaro (2007), estas condiciones observadas en el Atlántico Tropical favorecen la convección profunda, en donde anomalías positivas (negativas) de TSM en esta región coinciden con una mayor (menor) área de la piscina de aguas cálidas y mayor frecuencia de ciclones tropicales ${ }^{24}$. Lo anterior, es concordante con una menor PMNM, una mayor evaporación en la región y un mayor aporte de humedad a la atmósfera suprayacente. Por otro lado, durante la última parte de la estación lluviosa, es decir ASO, la variabilidad de la convección profunda en el Caribe está modulada tanto por la TSM en el Pacífico 
(a)

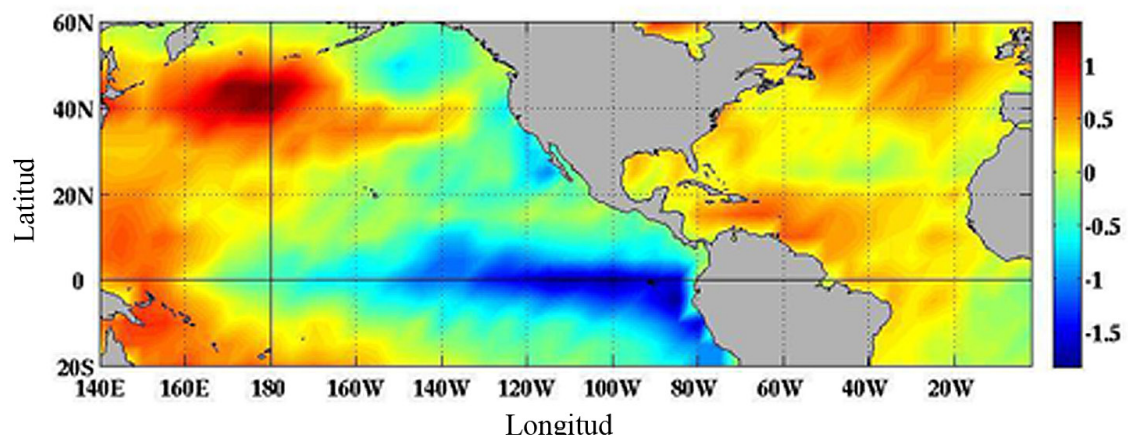

(b)

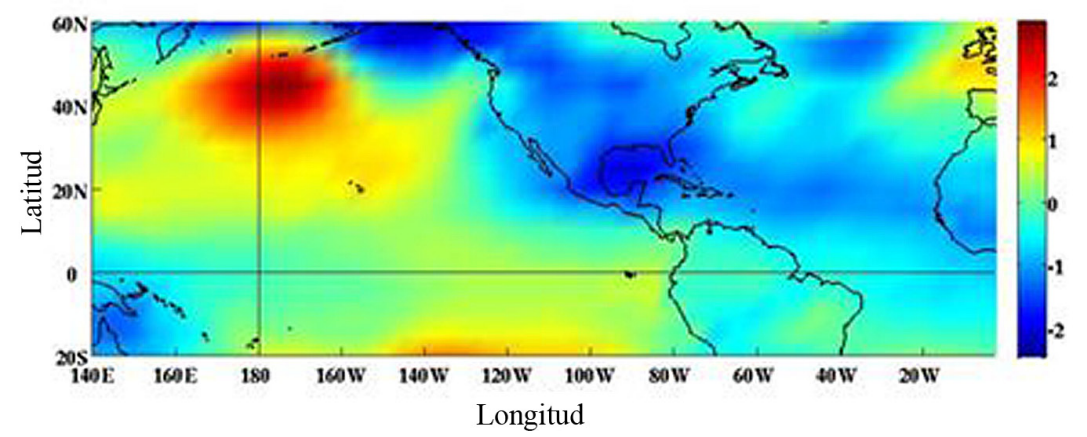

(c)

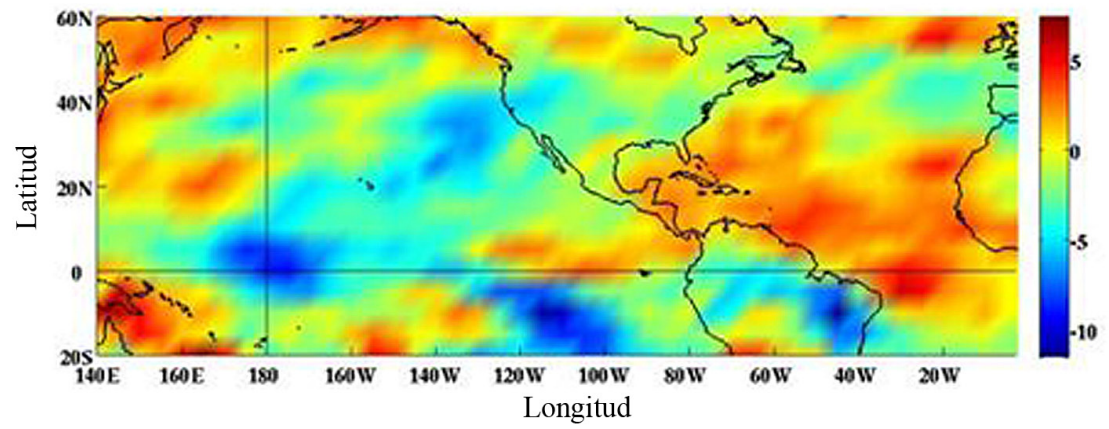

Figura 6. Mapas compuestos de anomalías de a) Temperatura Superficial del $\operatorname{Mar}\left({ }^{\circ} \mathrm{C}\right)$, b) Presión Atmosférica al Nivel del Mar (hPA) y c) Humedad Relativa (\%) en el nivel de $700 \mathrm{hPa}$. Los mapas consisten en la diferencia del promedio de aquellos años con mayor ocurrencia con respecto a los de menor ocurrencia durante el trimestre de agosto-setiembre-octubre. 
como en el Atlántico ecuatorial al modular la estructura de la cortante vertical del viento en la troposfera sobre el Atlántico Tropical Norte, en donde eventos cálidos (fríos) en el Pacífico ecuatorial aumentan (disminuyen) la cortante vertical en el Caribe, lo que trabaja en contra (a favor) de la convección profunda en esa región afectando la formación de ciclones tropicales ${ }^{25}$.

Para contrastar, el comportamiento en las curvas de frecuencias y ocurrencias de ciclones tropicales en el Caribe con los reportes de impactos por desastres causados por eventos de origen natural en Costa Rica, se extrajo el número anual de reportes de desastres de origen hidrometeorológico de la base de datos DesInventar ${ }^{26}$. La Fig. 7 muestra el total de los valores anuales encontrados y también aquellos registrados en presencia de un ciclón tropical en el Mar Caribe. La correlación de Pearson, entre estas series es de 0.81, con una significancia estadística superior al $99 \%{ }^{27}$. Se observó una marcada tendencia lineal en ambas series con un valor- $p$ asociado mucho menor a 0.010 . En la base de datos de DesInventar, los reportes de desastres aumentan a partir

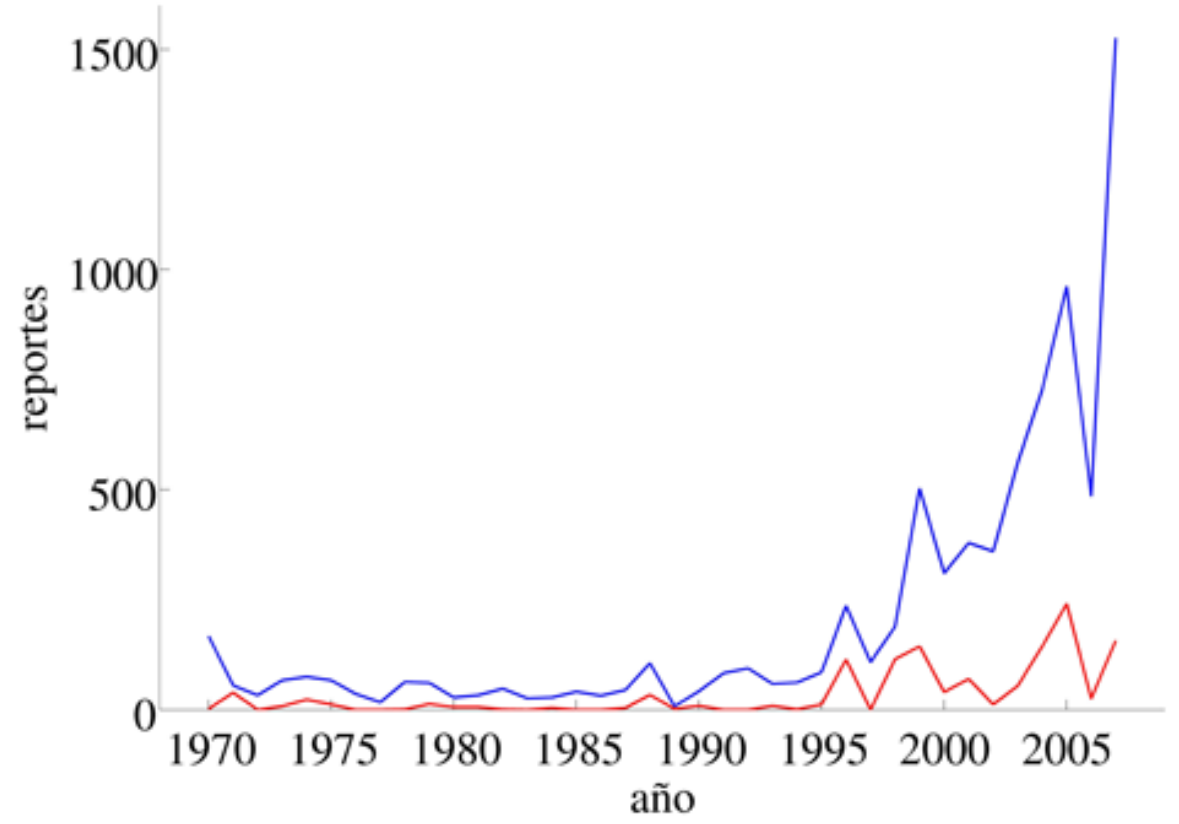

Figura 7. En azul, número anual de reportes de desastres de origen hidrometeorológico de la base de datos DesInventar y en rojo aquellos registrados durante la presencia de un ciclón tropical en el Mar Caribe. La tendencia lineal observada en ambas series fue de 18.77 y 3.02 reportes año ${ }^{-1}$, respectivamente, ambas con un valor-p mucho menor a 0.010 . 
de la segunda mitad de la década de 1990, probablemente, por el uso de una fuente de información oficial la Comisión Nacional de Emergencias de Costa Rica (CNE), en el periodo $1995-2007^{28}$. Por otra parte, aunque en Costa Rica se ha observado en los últimos años que el porcentaje de personas viviendo en condición de pobreza ha permanecido aproximadamente constante, fluctuando alrededor de un $20 \%{ }^{29}$, también hubo un incremento de los hogares que no satisfacen las necesidades básicas, más aquellos viviendo en extrema pobreza. Según las estadísticas del Programa, en 1990 los hogares en condición de pobreza sumaban 131981 (27.1\% del total), mientras que en el 2007 estos fueron 186 $461(16.7 \%)$ y $195521(17.7 \%)$ en el 2008, para un crecimiento en este rubro de 41 y $48 \%$ con respecto a 1990, respectivamente. En términos absolutos, el número de personas viviendo en condición de pobreza ha aumentado, lo que podría estar relacionado con un mayor número de personas vulnerables ante el impacto de algún evento de tipo hidrometeorológico que puede ocasionar un reporte de desastre. Sin embargo, la pobreza no es el único factor que determina la vulnerabilidad. Más del $70 \%$ de los reportes de desastres de la base de datos están asociados a eventos de origen hidrometeorológico, en particular inundaciones y deslizamientos (ver: http://online.desinventar.org/, para más detalles, última visita 21/01/2010). Estos se presentan principalmente entre los meses de mayo y noviembre, lo que coincide con la estación lluviosa en la Vertiente del Pacífico y la temporada de huracanes en el Atlántico Norte (Taylor y Alfaro, 2005; Alfaro, 2002). Todas las provincias tienen uno o varios cantones o municipios afectados de manera recurrente por algún evento natural, socio-natural o antrópico. Las inundaciones y los deslizamientos registran más del $40 \%$ de las muertes, seguidos de la actividad volcánica y los incendios. Los sismos $(60 \%)$, las inundaciones $(21 \%)$ e incendios $(12 \%)$ tienen registrado más del $90 \%$ de las viviendas destruidas ${ }^{30}$

Las tendencias mostradas por los reportes extraídos de DesInventar, contrastan con los valores encontrados para la frecuencia anual de ciclones tropicales en el Caribe (Fig. 2) y con la ocurrencia de los mismos cerca de la costa (Fig. 3), en donde ambas series no tienen tendencias lineales significativas asociadas. Durante los años de mayor ocurrencia de ciclones cerca de América Central se registraron en total 1880 casos ligados a eventos hidrometeorológicos, mientras que, durante los años de menor ocurrencia, esta cifra fue de apenas 161 casos. Para aquellos casos ocurridos durante la presencia de ciclones tropicales en el Mar Caribe, estas cifras disminuyeron apreciablemente y fueron de 593 y 3 , respectivamente.

Los casos mostrados en la Fig. 7 fueron agrupados por provincias (Fig. 8). Nótese que San José es la que presenta valores más altos en ambas situaciones con 562 y 212, seguido de la provincia de Puntarenas. El tercer lugar de la Fig. 8a lo ocupa la provincia de Limón, lo cual se debe a que se están considerando también los reportes de desastres 
(a)

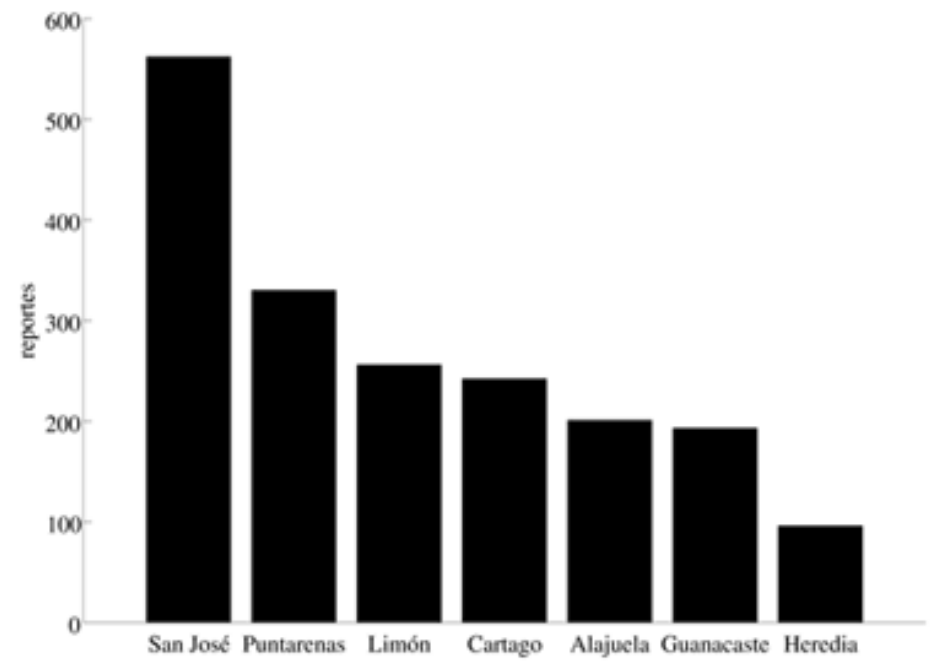

(b)

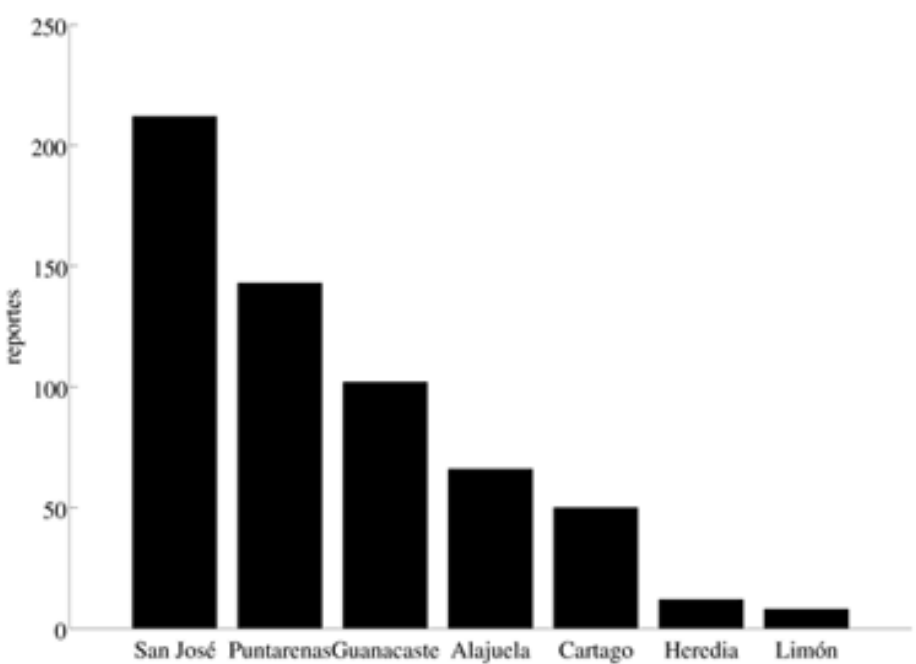

Figura 8. Reportes de desastres por provincia según la base de datos DesInventar asociados a: a) impacto de un evento hidrometeorológico y b) impacto de un evento hidrometeorológico durante la presencia de ciclones tropicales en el Caribe. Lo anterior durante los años de mayor ocurrencia de ciclones tropicales cerca de la costa caribe de América Central según la Fig. 4. 
asociados con los frentes fríos invernales que alcanzan Costa Rica típicamente entre los meses de noviembre y marzo, normalmente asociados a los "temporales" de invierno boreal $^{31}$ y que afectan sobre todo a la provincia de Limón. Cuando se analizan los reportes realizados únicamente durante la presencia de ciclones tropicales en el Caribe, que ocurren mayormente durante el verano y otoño boreales, esta provincia pasa a ocupar el último lugar (Fig. 8b). Lo anterior concuerda con los registros de los impactos de estos eventos en Costa Rica, la mayoría de los cuales se observa en la vertiente pacífica del país (llamados también efectos "indirectos"), provocados por la circulación inducida en el istmo por el ciclón ${ }^{32}$.

\begin{tabular}{cccccc}
\multicolumn{3}{c}{ Años de menor ocurrencia } & \multicolumn{3}{c}{ Años de mayor ocurrencia } \\
$\begin{array}{c}\text { A mérica } \\
\text { Central }\end{array}$ & Antillas & $\begin{array}{c}\text { Golfo de } \\
\text { México }\end{array}$ & $\begin{array}{c}\text { A mérica } \\
\text { Central }\end{array}$ & Antillas & $\begin{array}{c}\text { Golfo de } \\
\text { México }\end{array}$ \\
1976 & 1983 & 1981 & 2007 & 1975 & 1979 \\
1983 & 1977 & 1991 & 2001 & 1970 & 1971 \\
1984 & 1986 & 1986 & 1995 & 2006 & 1985 \\
1986 & 1992 & 2006 & 1996 & 2004 & 2003 \\
1992 & 1978 & 1976 & 2000 & 2005 & 1998 \\
1997 & 1997 & 1984 & 1969 & 2007 & 2002 \\
1981 & 1993 & 1992 & 1971 & 1981 & 2005 \\
1985 & 1976 & 1975 & 2005 & 1979 & 1995
\end{tabular}

Tabla 1. Años con menores (izquierda) y mayores (derecha) posiciones registradas dentro de un radio de $3^{\circ}$ de algún punto de la costa caribe de América Central, las Antillas y del Golfo de México. El orden de los años está de acuerdo al número de posiciones encontradas, es decir de menor a mayor para cada región en particular. Los años se ordenaron en forma creciente (de menor a mayor) con respecto al número de posiciones encontradas.

Los reportes de eventos ligados a la presencia de algún ciclón tropical en el Caribe durante los años de mayor ocurrencia, fueron agrupados también por cantón o municipio y su siguiente unidad administrativa más pequeña, el distrito (Tablas 2 y 3). Los dos cantones que presentaron mayores reportes fueron Desamparados en la provincia de San José y el cantón Central de la provincia de Puntarenas con 29 reportes cada uno (Tabla 2). Los dos distritos con mayores reportes fueron Santiago y San Marcos, ubicados en la provincia de San José, seguidos de Parrita, en el litoral central de la provincia de Puntarenas. Los reportes observados en estos distritos fueron 15, 14 y 13, respectivamente (Tabla 3). Cabe destacar, que todas las unidades administrativas descritas en las Tablas 2 y 3 se encuentran ubicadas en la Vertiente del Pacífico de Costa Rica (Fig. 9), concordando con el hecho de que la mayoría de los impactos sobre el país son de tipo "indirecto" 


\begin{tabular}{|c|c|c|c|c|c|c|}
\hline Número & Provincia & Cantón & Reportes & IDS 2007 & $\begin{array}{l}\text { Posición } \\
\text { según IDS }\end{array}$ & Categoría \\
\hline 1 & San José & Desamparados & 29 & 49.7 & 38 & $\mathrm{~N}$ \\
\hline 2 & Puntarenas & Puntarenas & 29 & 26.7 & 62 & $\mathrm{BN}$ \\
\hline 3 & San José & Puriscal & 27 & 48.3 & 43 & $\mathrm{~N}$ \\
\hline 4 & San José & Pérez Zeledón & 26 & 36.8 & 55 & $\mathrm{BN}$ \\
\hline 5 & Guanacaste & Santa Cruz & 26 & 57.4 & 24 & AN \\
\hline 6 & Guanacaste & Nicoya & 25 & 39.5 & 50 & $\mathrm{BN}$ \\
\hline 7 & Guanacaste & Nandayure & 24 & 37.5 & 54 & $\mathrm{BN}$ \\
\hline 8 & San José & Tarrazú & 23 & 30.4 & 58 & $\mathrm{BN}$ \\
\hline 9 & San José & León Cortés Castro & 21 & 36.2 & 56 & $\mathrm{BN}$ \\
\hline 10 & Cartago & La Unión & 20 & 52.9 & 30 & AN \\
\hline
\end{tabular}

Tabla 2. Reportes de eventos asociados a la presencia de algún ciclón tropical en el Caribe durante los años de mayores ocurrencias cerca de la costa Caribe de América Central (Fig. 4) para los primeros 10 cantones o municipios (columna 4). Se muestra también el valor y la posición observada del Índice de Desarrollo Social (IDS) del 2007 en dicho cantón con respecto al total del país de 81 (columnas 5 y 6). Las posiciones se ordenaron de menor a mayor y se agruparon en cinco categorías (columna 7), a saber, muy por abajo de lo normal $(\mathrm{MBN})$, abajo de lo normal $(\mathrm{BN})$, normal $(\mathrm{N})$, arriba de lo normal (AN) y muy por arriba de lo normal (MAN). La columna 2 muestra la provincia en la que se ubica dicho cantón o municipio. El número de identificación del cantón de la columna 1 se graficó en azul en la Fig. 9 para mostrar su ubicación geográfica.

$\begin{array}{cccccccc}\text { Número } & \text { Provincia } & \text { Cantón } & \text { Distrito } & \text { Reportes } & \begin{array}{c}\text { IDS } \\ \mathbf{2 0 0 7}\end{array} & \begin{array}{c}\text { Posición } \\ \text { según IDS }\end{array} & \text { Categoría } \\ 1 & \text { San José } & \text { Puriscal } & \text { Santiago } & 15 & 67.3 & 70 & \text { MAN } \\ 2 & \text { San José } & \text { Tarrazú } & \text { San Marcos } & 14 & 50.1 & 294 & \text { BN } \\ 3 & \text { Puntarenas } & \text { Parrita } & \text { Parrita } & 13 & 49.8 & 298 & \text { BN } \\ 4 & \text { Guanacaste } & \text { Nandayure } & \text { Bejuco } & 12 & 37.4 & 419 & \text { MBN } \\ 5 & \text { Guanacaste } & \text { Santa Cruz } & \text { Santa Cruz } & 12 & 65 & 101 & \text { AN } \\ 6 & \text { Puntarenas } & \text { Corredores } & \text { Corredor } & 12 & 40.6 & 388 & \text { MBN } \\ 7 & \text { Cartago } & \text { La Unión } & \text { Río Azul } & 10 & 51.5 & 276 & \text { N } \\ 8 & \text { Puntarenas } & \text { Golfito } & \text { Golfito } & 10 & 39 & 404 & \text { MBN } \\ 9 & \text { Puntarenas } & \text { Aguirre } & \text { Quepos } & 10 & 52.8 & 262 & \text { N } \\ 10 & \text { Alajuela } & \text { Alajuela } & \text { Alajuela } & 9 & 59.9 & 168 & \text { AN } \\ 11 & \text { Puntarenas } & \text { Osa } & \text { Cortés } & 9 & 53.9 & 245 & \text { N } \\ 12 & \text { San José } & \text { Zeledón } & \text { General } & 9 & 57.7 & 192 & \text { N }\end{array}$

Tabla 3. Reportes de eventos asociados a la presencia de algún ciclón tropical en el Caribe durante los años de mayores ocurrencias cerca de la costa Caribe de América Central (Fig. 4) para los primeros 12 distritos (columna 5). Se muestra también el valor y la posición observada del Índice de Desarrollo Social (IDS) del 2007 en dicho distrito con respecto al total del país de 469 (columnas 6 y 7). Las posiciones se ordenaron de menor a mayor y se agruparon en cinco categorías (columna 8), a saber, muy por abajo de lo normal (MBN), abajo de lo normal (BN), normal (N), arriba de lo normal (AN) y muy por arriba de lo normal (MAN). Las columna 2 y 3 muestran la provincia y el cantón o municipio en la que se ubica dicho distrito. El número de identificación del distrito de la columna 1 se graficó en rojo en la Fig. 9 para mostrar su ubicación geográfica. 
Para contrastar el valor del número de reportes en los diferentes cantones y distritos del país con alguna variable social, se tabuló el Índice de Desarrollo Social (IDS) elaborado por MIDEPLAN en 2007 para estas unidades administrativas (Tablas 2 y 3). Se observa en la Tabla 2 que 8 de los 10 cantones presentaron un IDS con categoría normal $(\mathrm{N})$, bajo lo normal $(\mathrm{BN})$ o muy por debajo de lo normal MBN y de la Tabla 3 que 9 de los 12 distritos presentaron un IDS en dichas categorías. Lo anterior sugiere que la mayoría de las unidades administrativas presentadas en las Tablas 2 y 3 no muestran un IDS alto o muy alto; sin embargo, esto requiere un estudio posterior debido a que el IDS analizado corresponde al año 2007 y el número de eventos reportados corresponde al periodo 1970-2007; además, situaciones particulares en unidades administrativas más pequeñas a las consideradas podrían elevar el IDS de algún cantón o distrito específico. Al comparar nuestros resultados con los presentados por el Instituto Mixto de Ayuda Social ${ }^{33}$, se nota que los cantones de Desamparados, central de Puntarenas y Pérez Zeledón, también se ubican entre los primeros cantones con mayor número de familias en pobreza básica y en pobreza extrema.

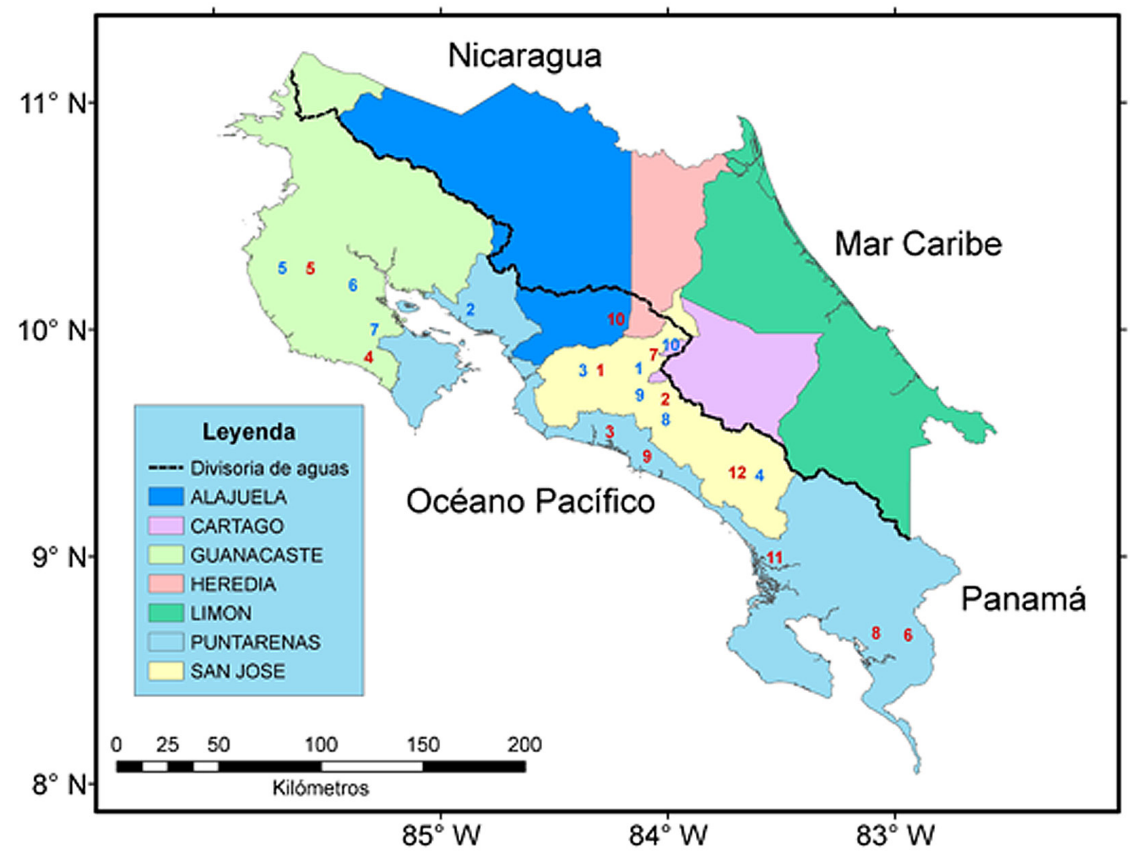

Figura 9. Ubicación geográfica de los cantones o municipios (números en azul) y de los distritos (números en rojo) de acuerdo a las primeras columnas de las Tablas 2 y 3. La línea negra gruesa muestra la divisoria de aguas entre aquellas cuencas que vierten al océano Pacífico y aquellas que lo hacen al Mar Caribe. 
La Tabla 4 muestra las correlaciones de Pearson entre las series de tiempo de las Figs. 2, 3 y 7. Se observa, que todas las correlaciones calculadas fueron positivas y todas las significancias estadísticas de los eventos reportados durante la presencia de ciclones tropicales son superiores al 99\% (columna derecha). Lo anterior es importante ya que describe una covariabilidad significativa de las series, a pesar que no concuerden necesariamente con el comportamiento y/o significancia de su tendencia lineal.Para ambas series de tiempo asociadas a los desastres producidos por eventos hidrometeorológicos, la mayor correlación se observó con la serie de tiempo asociada a la frecuencia de huracanes intensos, sin embargo los huracanes intensos fueron solamente el 11\% del total de ciclones tropicales en el periodo de estudio (Fig. 2). Lo anterior es relevante ya que el impacto del ciclón sobre las comunidades en Costa Rica no está necesariamente relacionado con su intensidad, sino que también en función de su posición y permanencia en la cuenca con respecto al sitio de interés. Un ejemplo reciente de lo anterior fue la depresión tropical No. 16 en el año 2008 que ocasionó condiciones de fuerte temporal en el litoral pacífico del 12 al 17 de octubre. Se reportaron 486 comunidades afectadas con inundaciones, deslizamientos, afectación en puentes, carreteras, infraestructura y otros. Hubo 18533 personas afectadas directamente por inundaciones y deslizamientos, para un total de 92655 personas afectadas ${ }^{34}$.

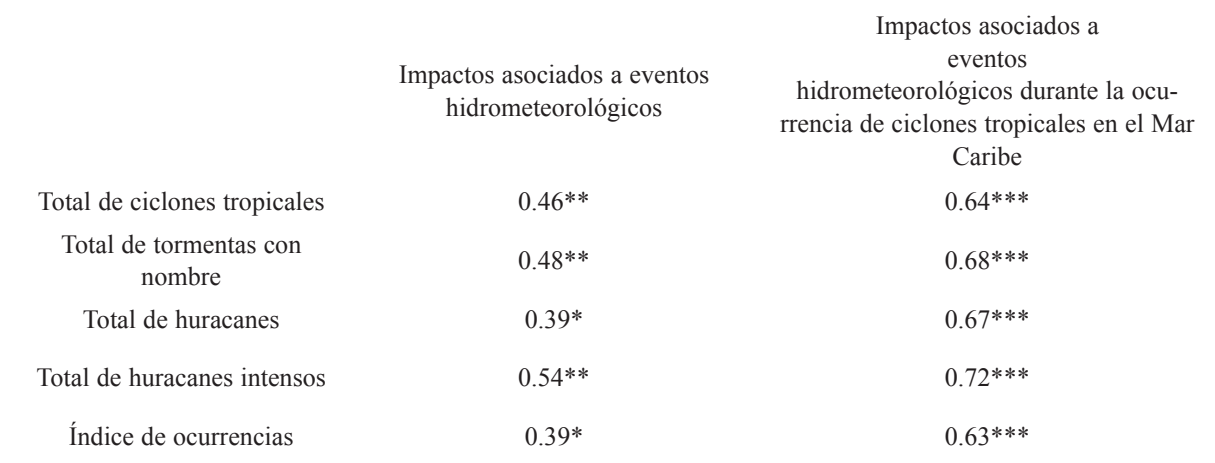

Tabla 4. Correlaciones de Pearson entre las series de tiempo de las Figs. 2, 3 y 7. Su significancia estadística fue calculada de acuerdo a Davis (1976), en donde $\alpha=0.01=>* * *, 0.05=>* *, 0.10 \Rightarrow *$, para una significancia estadística de 99,95 y $90 \%$, respectivamente.

Con el fin de hacer un análisis exploratorio entre el índice de ocurrencias presentado en la Fig. 3 y alguna variable macroeconómica, se presenta en la Fig. 10 el diagrama de dispersión de la tasa de variación del PIB a precios de mercado vrs. el índice de ocurrencias. De la figura se nota que el 35\% de las veces (14 casos) en que el índice de ocurrencias se observó por arriba (abajo) de su mediana, la tasa de variación se observó por abajo (arriba) de su respectiva mediana. Nótese 
también que, la probabilidad de observar un índice de ocurrencia por arriba (abajo) de su mediana en concordancia con una tasa de variación también por arriba (abajo) de su mediana fue menor, con un 15\% (6 eventos) en ambos casos. Esta relación merece un estudio posterior, ya que las variaciones del PIB no sólo se ven influenciadas por variables climáticas no asociadas a los ciclones tropicales, sino también por distintos factores externos como los mercados internacionales que no necesariamente están relacionadas con la variabilidad climática, por mencionar únicamente uno de ellos.

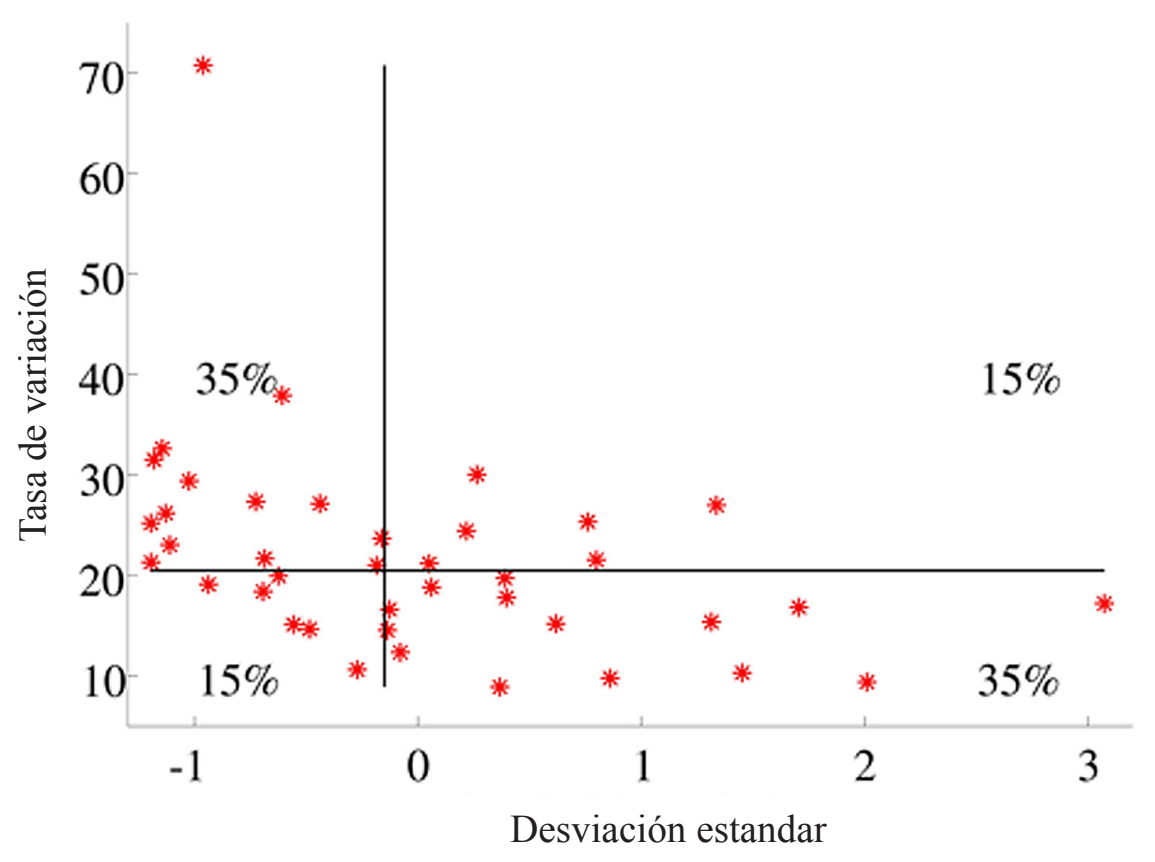

Figura 10. Diagrama de dispersión de la tasa de variación del PIB a precios de mercado versus el índice de ocurrencias presentado en la Fig. 3. La línea sólida horizontal y vertical representa la mediana de cada una de las variables.

\section{CONCLUSIONES}

No se encontró una tendencia lineal positiva estadísticamente significativa en el número anual del total de ciclones tropicales observados en el Mar Caribe en la base de datos HURDAT, ni tampoco en la suma de las posiciones anuales registradas dentro de un radio de $7^{\circ}$ de algún punto de la costa Caribe de América Central para los 40 años del periodo 1968-2007 (Figs. 2 y 3). Sin embargo, si se hayó una tendencia lineal positiva y estadísticamente significativa en el número anual de huracanes intensos, o sea, aquellos que registraron una categoría 3 o 
mayor, en el Mar Caribe de acuerdo a la escala de Saffir-Simpson. Estos últimos representaron el 11\% del total de los ciclones considerados en el estudio.

El impacto de un ciclón en las comunidades en Costa Rica no está necesariamente relacionado con su intensidad, sino que también está en función de su posición y permanencia en la cuenca con respecto al sitio de interés o velocidad de avance ${ }^{35}$. Un ejemplo de lo anterior fue la depresión tropical No. 16 en el año 2008 que ocasionó condiciones de fuerte temporal en el litoral pacífico del 12 al 17 de octubre, donde se reportaron muchas comunidades afectadas con inundaciones, principalmente en la provincia de Guanacaste (Fig. 9). Los principales reportes de impactos fueron en deslizamientos, afectación en puentes, carreteras e infraestructura. También se reportaron inundaciones importantes en Guatemala, Honduras, El Salvador y Nicaragua ${ }^{36}$.

Los meses en los cuales es más probable la ocurrencia de ciclones tropicales cerca de la costa caribe de América Central fueron agosto-setiembre-octubre (69\%, Fig. 5). Al comparar la distribución espacial de diferentes variables geofísicas durante los años de mayor ocurrencia de ciclones tropicales cerca de América Central, con respecto a aquellos años de menor ocurrencia (Fig. 3), se notó que durante este trimestre se presentaron en el Atlántico Tropical Norte condiciones de anomalías positivas de temperatura superficial del mar y humedad relativa en el nivel de $700 \mathrm{hPa}$; y negativas en la presión media al nivel del mar (Fig. 6). Todo lo anterior favorece la convección profunda y fomenta la formación de ciclones tropicales. Por otra parte, durante este trimestre, se observaron también anomalías negativas en la región del Pacífico ecuatorial, lo anterior también favorece la convección profunda y la formación de ciclones tropicales en el Atlántico Tropical al disminuir la cortante vertical del viento sobre esa región ${ }^{37}$.

Al estudiar, el número anual de desastres asociados a eventos hidrometeorológicos en la base de datos DesInventar, se encontró una tendencia lineal positiva y estadísticamente significativa en el período 1970-2007 (Fig. 7). Este mismo resultado se halló para aquellos reportes realizados durante la presencia de algún ciclón tropical en el Mar Caribe. La razón de lo anterior no es clara y podría asociarse a un mejor registro de los desastres por una fuente de información oficial, la Comisión Nacional de Emergencias de Costa Rica, en el periodo 1995 - 2007. Por otra parte, aunque en Costa Rica se ha observado en los últimos años que el porcentaje de personas viviendo en condición de pobreza ha permanecido aproximadamente constante ${ }^{38}$, también hubo un aumento de los hogares que no satisfacen las necesidades básicas, más aquellos viviendo en extrema pobreza. En términos absolutos, el número de personas viviendo en condición de pobreza ha aumentado, lo que podría estar relacionado con un mayor número de personas vulnerables ante el impacto de algún evento de tipo hidrometeorológico que puede generar un reporte de desastre. Por lo anterior, es recomendable un estudio posterior 
que profundice en este tema, e incluya además otras bases de datos sobre desastres de origen natural, lo que permitiría verificar este resultado.

Todas las unidades administrativas en el nivel cantonal (municipios) y distrital que reportaron mayor cantidad de desastres en presencia de algún ciclón tropical en el Mar Caribe, se ubicaron en la Vertiente del Pacífico de Costa Rica (Tablas 2 y 3 y Fig. 9). Lo anterior confirma que la mayoría de los efectos sufridos en el país provocados por estos eventos son los llamados efectos "indirectos", es decir, aquellos inducidos por la interacción del flujo del oeste con la topografía local, lo cual está asociado también a la ocurrencia de los llamados "temporales" en la Vertiente del Pacífico de Costa Rica, con lluvia continua sobre este vertiente, en general relacionado a nubosidad de tipo estratiforme ${ }^{39}$. Por último, al analizar el Índice de Desarrollo Social del año 2007 para todas las unidades administrativas de Costa Rica y agruparlas en cinco categorías; a saber, muy por debajo de lo normal $(\mathrm{MBN})$, bajo lo normal $(\mathrm{BN})$, normal $(\mathrm{N})$, arriba de lo normal (AN) y muy por arriba de lo normal (MAN), se encontró que la mayoría de las unidades descritas en la Fig. 9 se ubicaron en las categorías N, BN o MBN (Tablas 2 y 3 ).

\section{AGRADECIMIENTOS}

Este trabajo se realizó gracias a los proyectos CRN2050-IAI y 805-A7002, A8-606, A9-532-UCR. También se agradece al Programa de Estudios Sociales de la Ciencia, la Técnica y el Medio Ambiente, 805-A4-906-UCR. Se agradece a Tito Maldonado por su colaboración en el procesamiento de algunos de los datos y figuras. A David Enfield, Adriana Bonilla, Berny Fallas, Xinia Soto, Luis F. Alvarado y Omar Lizano por sus comentarios y sugerencias durante la elaboración del trabajo.

\section{REFERENCIAS}

Alfaro, E. "Escenarios climáticos para temporadas con alto y bajo número de huracanes en el Atlántico", Revista de Climatología 7 (2007): 1-13.

Alfaro E. "Some Characteristics of the Annual Precipitation Cycle in Central America and their Relationships with its Surrounding Tropical Oceans", Tópicos Meteorológicos y Oceanográficos 9, no.2 (2002): 88-103.

Alvarado, L. y E. Alfaro. "Frecuencia de los ciclones tropicales que afectaron a Costa Rica durante el siglo XX”, Tópicos Meteorológico y Oceanográficos 10, no.1 (2003): 1-11.

Amador, J.A. y A. "Ciclones tropicales y sociedad: Una aproximación al enfoque científico de estos fenómenos atmosféricos como referente para la investigación social en desastres", en Concepciones y Representaciones de la Naturaleza y la 
Ciencia en América Latina, editado por R.Viales, J. Amador y F.J. Solano. San José: Editorial de la Universidad de Costa Rica, 2009, 159-179.

Banichevich, A. y O. Lizano. "Interconexión a nivel ciclónico-atmosférico entre el Caribe y el Pacífico centroamericanos". Revista de Biología Tropical 46, Supl.5 (1998): 5, 9-22.

Bender, M.A., T.R. Knutson, R.E. Tuleya, J.J. Sirutis, G.A. Vecchi, S.T. Garner, y I.M. Held. "Modeled Impact of Anthropogenic Warming on the Frequency of Intense Atlantic Hurricanes". Science 327 (2010): 454-458.

Bengtsson, L., K.I. Hodges, M. Esch, N. Keenlyside, L. Kornblueh, J.-L. Luo, y T. Yamagata: "How tropical cyclones may change in a warmer climate". Tellus $59 \mathrm{~A}$ (2007): 539-561.

Beven II, J.L., L.A. Avila, E.S. Blake, D.P. Brown, J.L. Franklin, R.D. Knabb, R.J. Pasch, F.R. Rhome, y S.R. Stewart: "Annual Summary: Atlantic Hurricane Season of 2005". Monthly Weather Review 136, no.3: (2008): 1131-1141.

Bonilla, A. y J.A. Amador. "El temporal de enero 2000: sus características e impactos socio-económicos sobre las comunidades próximas a la cuenca de la Laguna de Arenal y de la región Húetar Norte", en Concepciones y Representaciones de la Naturaleza y la Ciencia en América Latina, editado por R. Viales, J.A. Amador y F.J. Solano. San José: Editorial de la Universidad de Costa Rica, 2009, 243-253.

Chinchilla, G. "Comentario meteorológico de Octubre de 2008". Boletín Meteorológico. XXXIII, Octubre 2008 [citado el 22 de enero de 2010]. Instituto Meteorológico Nacional. San José, Costa Rica: 2-13. Disponible en http://www. imn.ac.cr/boletin meteo/historial/2008/BMET102008.pdf.

Davis, R.: "Predictability of sea surface temperature and sea level pressure anomalies over the North Pacific Ocean”. Journal of Physical Oceanography 6, (1976): 249-266.

De Maria, M., J.A. Knaff, y B.H. Connell. "A tropical cyclone genesis parameter for the Tropical Atlantic”. Weather Forecast 16, (2001): 219-233.

Fallas, J.C y R. Oviedo. “Temporales”. Cap. III. En: Fenómenos atmosféricos y cambio climático, visión centroamericana. Instituto Meteorológico Nacional, San José, Costa Rica, (2003): 38.

Goldenberg, S., C. Landsea, A. Mestas-Nuñez, y W. Gray. " The recent increase in Atlantic hurricane activity: Causes and Implications". Science, 293 (2001): 474-479.

Gutowski Jr., W. et al. "Causes of Observed Changes in Extremes and Projections of Future Changes, Chapter 3. En: Weather and Climate Extremes in a Changing Climate, Regions of Focus: North America, Hawaii, Caribbean, and U.S. Pacific Islands. Synthesis and Assessment Product 3.3. Report by the U.S. Climate Change Science Program and the Subcommittee on Global Change Research. 2008, 81-116.

Hobgood, J. "Tropical Cyclones". En Encyclopedia of World Climatology, editado por J. Oliver. Springer, Netherlands, 2005: 750-755.

Kalnay, E., et al. "The NCEP/NCAR Reanalysis 40-year Project". Bulletin of the 
American Meteorological Society 77 (1996): 437-471.

Kaplan, A., M.A. Cane, Y. Kushnir, A.C. Clement, M.B. Blumenthal, y B. Rajagopalan,: "Analysis of global sea surface temperatures 1856-1991". Journal of Geophysical Research 103 (1998): 18567-18589.

Klotzbach, P. J. "Trends in global tropical cyclone activity over the past twenty years (1986-2005)". Geophysical Research Letters, 33 (2006): L10805, doi:10.1029/2006GL025881.

Knutson, T.R., J.J. Sirutis, S.T. Garner, G.A. Vecchi, y I.M. Held, "Simulated reduction in Atlantic hurricane frequency under twenty-first-century warming conditions". Nature Geoscience 1, no.7 (2008): 359-364.

Kossin, J. P., K. R. Knapp, D. J. Vimont, R. J. Murnane, y B. A. Harper. "A globally consistent reanalysis of hurricane variability and trends". Geophysical Research Letters 34, (2007): L04815, doi:10.1029/2006GL028836.

Kunkel, K. "Observed Changes in Weather and Climate Extremes", Chapter 2. En: Weather and Climate Extremes in a Changing Climate, Regions of Focus: North America, Hawaii, Caribbean, and U.S. Pacific Islands. Synthesis and Assessment Product 3.3. Report by the U.S. Climate Change Science Program and the Subcommittee on Global Change Research. 2008, 35-80.

Landsea, C.W., G. A.Vecchi, L. Bengtsson, y T. R. Knutson.“ Impact of duration thresholds on Atlantic tropical cyclone counts". Journal of Climate, (2009): doi:10.1175/2009JCLI3034.1.

Landsea, C.W., B. Harper, K. Hoarau, y J. Knaff. "Can we detect trends in extreme tropical cyclones“. Science 313 (2006): 452-454.

Landsea. C.W., et al., 2004: "The Atlantic Hurricane database Re-analysis Project". En Hurricanes and Typhoons, past, present and future, editado por R. Murnane \& K. Liu, Columbia University Press, 2004, 177-221.

Latif, M., N. Keenlyside, and J. Bader. "Tropical sea surface temperature, vertical wind shear, and hurricane development". Geophysical Research Letters 34 (2007): L01710, doi:10.1029/2006GL027969.

Liu, K. "Uncovering Prehistoric Hurricane Activity". American Scientist 95(2007): $126-133$.

Lizano, O. G. "Un método manual para el pronóstico de oleaje durante huracanes en el Caribe adyacente a Costa Rica”. Tópicos Meteorológicos y Oceanográficos 3, no.1 (1996):11-17.

Lizano, O.G., y W. Fernández. “Algunas características de las tormentas tropicales $\mathrm{y}$ de los huracanes que atravesaron o se formaron en el Caribe adyacente a Costa Rica durante el periodo 1886-1988". Tópicos Meteorológico y Oceanográficos, 3, no.1 (1996): 3-10. 
MIDEPLAN (Ministerio de Planificación Nacional y Política Económica de Costa Rica, Área de análisis del desarrollo)."Indice de Desarrollo Social 2007". San José, Costa Rica. Enero, 2007 [citado el 22 de enero del 2010]. Disponible en http://www.mideplan.go.cr/sides/social/indx10.htm

Neumann, C., B. Jarvinen, C. McAdie, y J. Elms. "Tropical Cyclones of the North Atlantic Ocean, 1871-1992", Prepared by the National Climatic Data Center, Asheville, NC, in cooperation with the National Hurricane Center, Coral Gables, FL. 1993:193.

Pielke, R., J. Rubiera, C. Landsea, M. Fernandez, y R. Klein. "Hurricane vulnerability in Latin America and the Caribbean: Normalized damage and loss potentials". Natural Hazards Review, (1993):101-114.

Programa Estado de la Nación. "Decimoquinto Informe Estado de la Nación en Desarrollo Humano Sostenible (2008”). Resumen. Imprenta LIL S.A. San José, Costa Rica. (2009).[citado el 22 de enero de 2010]: Disponible en http:// www.estadonacion.or.cr/

Reynolds, R., y T. Smith. "Improved global sea surface temperature analyses using optimum interpolation". Journal of Climate 7 (

Saunders, M.A., y A.S. Lea. "Large contribution of sea surface warming to recent increase in Atlantic hurricane activity". Nature 451 (2008):557-560.

Solano, F., E. Alfaro, y A. Quesada. "Impacto de los Ciclones Tropicales del Atlántico en Centroamérica, Temporada de 1969". Sometido a Revista Diálogos (2010): En revisión.

Taylor, M., y E. Alfaro E."Climate of Central America and the Caribbean". En Encyclopedia of World Climatology, editado por J. Oliver. Springer, Netherlands, 2005: 183-189.

Velásquez, A., y C. Rosales. "Escudriñando en los desastres a todas las escalas. Concepción, metodología y análisis de desastres en América Latina utilizando DesInventar". Red de Estudios Sociales en Prevención de Desastres en América Latina - LA RED. Cali, Colombia. 1999 [citado el 21 de enero de 2010]: Disponible en http://www.desinventar.org/es/projectos/lared/escudrinando

Wang, C., D. Enfield, S. Lee, y C. Landsea."Influences of the Atlantic warm pool on western hemisphere summer rainfall and Atlantic hurricanes". Journal of Climate 19 (2006): 3011-3028.

Wang, C., y S.-K. Lee. "Co-variability of tropical cyclones in the North Atlantic and the eastern North Pacific". Geophysical Research Letters 36 (2009): L24702, doi:10.1029/2009GL041469.

Woodruff, J. D., J. P. Donnelly, K. Emanuel, y P. Lane. “Assessing sedimentary records of paleohurricane activity using modeled hurricane climatology". Geochemistry, Geophysics and Geosystems. 9, (2008): Q09V10, doi:10.1029/2008GC002043. 


\section{CITAS Y NOTAS}

1 (e.g. Alfaro, 2007; Alvarado y Alfaro, 2003; Banichevich y Lizano, 1998; Lizano y Fernández, 1996)

2 (Amador y Bonilla, 2009)

3 (e.g. Gutowski, Jr., et al., 2008).

4 (e.g. Bender et al., 2010; Knutson et al., 2008; Bengtsson et al., 2007)

5 (e. g. Landsea et al., 2009; Kunkel, 2008; Kossin et al., 2007; Landsea et al., 2006)

(e.g. Woodruff, et al., 2008; Liu, 2007)

(e.g. Knutson et al., 2008; Saunders y Lea, 2008; Goldenberg et al., 2001)

8 (Amador y Bonilla, 2009; Bonilla y Amador, 2009; Pielke et al., 1993)

9 (Landsea et al. 2009; 2004)

10 http://www.aoml.noaa.gov/hrd/hurdat/ (última visita el 18 de enero de 2010)

11 De acuerdo a la clasificación empleada en este trabajo la Perturbación Tropical se define como una zona de inestabilidad atmosférica asociada a la existencia de un área de baja presión, la cual propicia la generación incipiente de vientos convergentes cuya organización eventual provoca el desarrollo de una depresión tropical. Para la Depresión Tropical, los vientos se incrementan en la superficie, producto de la existencia de una zona de baja presión. Dichos vientos alcanzan una velocidad sostenida menor o igual a 62 kilómetros por hora. En una Tormenta Tropical, el incremento continuo de los vientos provoca que éstos alcancen velocidades sostenidas entre los 63 y $118 \mathrm{~km} / \mathrm{h}$ y las nubes se distribuyen en forma de espiral. Cuando el ciclón alcanza esta intensidad se le asigna un nombre preestablecido por la Organización Meteorológica Mundial. Un Huracán es un ciclón tropical en el cual los vientos máximos sostenidos alcanzan o superan los $118.1 \mathrm{~km} / \mathrm{h}$. El área nubosa cubre una extensión entre los 500 y $900 \mathrm{~km}$ de diámetro, produciendo lluvias intensas. El ojo del huracán alcanza normalmente un diámetro que varía entre 24 y $40 \mathrm{~km}$, sin embargo, puede llegar hasta cerca de $100 \mathrm{~km}$. Los huracanes se clasifican como categoría: 1 si sus vientos máximos están entre 118.1 y 154, 2 entre 154.1 y 178, 3 entre 178.1 y 210,4 entre 210.1 y 250 y 5 si son mayores a $250 \mathrm{~km} \mathrm{hora}^{-1}$ (tomado de: imn.ac.cr)

12 (Hobgood, 2005)

13 (Alfaro 2007; Taylor y Alfaro 2005; Alvarado y Alfaro 2003; Lizano 1996)

14 (Hobgood, 2005; Taylor y Alfaro, 2005)

15 (Velásquez y Rosales, 1999)

16 Se seleccionaron los eventos y causas que tienen mayor relación con un fenómeno de índole hidrometeorológico. Las causas de orden hidrometeorológico serían en este caso: Lluvias, Inundación, Deslizamiento, El niño, Condición atmosférica, Depresión tropical, Desbordamiento, La Niña, Erosión, Inversión Térmica, Neblina, Sequia, Tempestad, Vendaval. Por otro lado, los eventos de índole hidrometeorológico serían: Inundación, Deslizamiento, Avenida, Lluvias, Vendaval, Marejada, Huracán, Sedimentación, Helada, Granizada, Sequía, Litoral, Aluvión, Alud, Tsunami, Licuación, Tormenta eléctrica, Nevada, Ola de calor, Tornado, Neblina.

17 (Solano et al., 2010)

18 (MIDEPLAN, 2007)

19 (Beven II et al., 2008)

20 (Taylor y Alfaro, 2005; Alvarado y Alfaro, 2003; Fallas y Oviedo, 2003)

21 (Fallas y Oviedo, 2003)

22 (Davis, 1976)

23 (Taylor y Alfaro, 2005)

24 (Saunders y Lea, 2008; Wang et al., 2006) 
(Wang y Lee, 2009; Latif et al., 2007; Alfaro, 2007; Alvarado y Alfaro, 2003)

(Velásquez y Rosales, 1999)

(Davis, 1976)

(ver: http://online.desinventar.org/, para más detalles, última visita 21/01/2010)

(Informe del Programa Estado de la Nación, 2009)

(ver: http://online.desinventar.org/, para más detalles, última visita 21/01/2010.

(Fallas y Oviedo, 2003)

(Alvarado y Alfaro, 2003; Fallas y Oviedo, 2003).

33 (ver http://www.imas.go.cr/perfil/sist_info/sipo/mapas_pobreza.html, última visita el $1 / 10 / 2010)$

34 (Chinchilla, 2008)

35 (Taylor y Alfaro, 2005; Alvarado y Alfaro, 2003; Lizano, 1996).

36 (Chinchilla, 2008)

37 (Wang y Lee, 2009; Latif et al., 2007; Alfaro, 2007; Hobgood, 2005; Alvarado y Alfaro, 2003; Lizano y Fernández, 1996)

38 (Programa Estado de la Nación, 2009)

39 (Fallas y Oviedo, 2003) 